Eur. J. Clin. Chem. Clin. Biochem.

Vol. 30, 1992, pp. 599-606

(C) 1992 Walter de Gruyter \& Co.

Berlin · New York

\title{
Liver Transplantation in a Boy with Acute Porphyria Due to Aminolaevulinate Dehydratase Deficiency
}

\author{
By Stig Thunell ${ }^{1}$, Ann Henrichson ${ }^{1}$, Ylva Floderus ${ }^{1}$, C. G. Groth ${ }^{2}$, B.-G. Eriksson ${ }^{2}$, Lisbeth Barkholt ${ }^{2}$, \\ A. Nemeth ${ }^{3}$, Birgitta Strandvik ${ }^{3 *}$, L. Eleborg ${ }^{4}$, L. Holmberg ${ }^{5}$ and J. Lundgren ${ }^{5}$ \\ 1 Department of Clinical Chemistry, St. Göran's Hospital, Stockholm, Sweden \\ ${ }^{2}$ Department of Transplantation Surgery, Huddinge Hospital, Karolinska Institute, Stockholm, Sweden \\ ${ }^{3}$ Department of Pediatrics, Huddinge Hospital, Karolinska Institute, Stockholm, Sweden \\ ${ }^{4}$ Department of Anesthesiology and Intensive Care, Huddinge Hospital, Karolinska Institute, Stockholm, Sweden \\ ${ }^{5}$ Department of Pediatrics, University of Lund, Lund Hospital, Lund, Sweden
}

(Received August 15, 1991/July 5, 1992)

Summary: The clinical and biochemical outcome of a liver transplantation in a seven-year-old boy with acute porphyria due to aminolaevulinate dehydratase deficiency is described. Before transplantation standard liver function tests were normal and the rationale for transplantation was that the new liver would reduce the metabolic disturbance and thus avert the porphyric symptoms. During the year after the transplantation, the functioning of the new liver has been excellent. Basal excretion of porphyrin and porphyrin precursors has remained unchanged but, with the new liver transplant the patient has been able to withstand several porphyrinogenic challenges without increasing the excretion. Episodes of neurological and respiratory crises may have been due to persistent porphyric vulnerability. Alternatively, two early attacks may have been caused by neurotoxic effects of cyclosporin in combination with the existing damage to nervous tissue.

\section{Introduction}

The acute hepatic porphyrias comprise a group of inborn errors of porphyrin metabolism characterized by the development, in some individuals, of a severe neuropsychiatric syndrome: the acute porphyric attack. The underlying mutations are localized in genes responsible for the production of different enzymes active in haem synthesis and give rise to a reduced activity of the affected enzyme and accumulation of metabolites. Four types of acute porphyria are recognized. Thus heterozygous deficiencies in porphobilinogen deaminase, coproporphyrinogen oxidase and protoporphyrinogen oxidase produce the clinical conditions of acute intermittent porphyria, hereditary coproporphyria and variegate porphyria, respectively.

\footnotetext{
* Now: Department of Pediatrics, University of Gothenburg, Eastern Hospital, Gothenburg, Sweden.
}

In aminolaevulinate dehydratase deficiency porphyria symptoms develop only in individuals who are homozygous or double-heterozygous for the condition in question $(1-5)$.

The four forms of acute porphyria, share the feature of 5-aminolaevulinate overproduction with two other metabolic conditions, tyrosinaemia and lead intoxication, which present with similar neurological symptoms. Thus, 5-aminolaevulinate or one of its metabolites, may play a role in the development of the typical neuropathy. Some experimental support for this hypothesis has been obtained $(6-10)$. The accumulation of 5-aminolaevulinate is obviously due to the inability of a decreased aminolaevulinate dehydratase activity to meet the need for haem synthesis. This situation may be precipitated by a variety of exogenous and endogenous agents which act by reducing the cellular free haem pool, the size of which 
regulates the activity of the haem pathway. Several drugs and biocides, as well as alcohol, stress, fasting and infection, have been implicated in this context. The mechanism seems to depend on a consumption of haem for production of cytochrome P450 or an induction of the haem degrading oxygenase enzyme, both of which are considered to be mainly or wholly confined to the liver (11).

The critical role assigned to the liver in acute porphyria suggests that exchange of the porphyric liver by a normal organ should eliminate the basis for the neurological crises. Thus, in the last few years a number of patients with liver-based metabolic diseases have been treated by liver transplantation, although standard liver function tests have been normal. In these cases, the aim of the procedure has been to cure the patient's disease by correction of the metabolic deficiency. The first such liver transplantation was performed in a patient with familial hypercholesterolaemia (12). More recently, several patients with primary hyperoxaluria have undergone such "metabolic" liver transplantation. Liver transplantations have also been performed in a few patients with erythropoietic protoporphyria due to imminent liver failure $(13-15)$. Liver exchange has, however, not been attempted in acute porphyria. The relentless clinical deterioration in some porphyric patients and the risk of fatal exacerbations does seem to justify liver exchange as a therapeutic option. In the present communication the clinical and biochemical outcomes of liver transplantation in a boy with acute porphyria due to aminolaevulinate dehydratase deficiency are described.

\section{Materials and Methods}

The methods used to analyse aminolaevulinic acid, porphobilinogen, porphyrins and porphyrin isomers in urine and faeces, and porphyrin, porphobilinogen deaminase activity and aminolaevulinate dehydratase activity in erythrocytes were described in a previous communication (4). Blood cyclosporin concentration was determined by radioimmunological assay, using a monoclonal specific antibody (Sandoz, Basel, Switzerland).

\section{Patient}

The clinical course and the diagnosis of aminolaevulinate dehydratase deficiency porphyria in our patient were described in a previous report (4). The diagnosis was based on the findings of an erythrocyte aminolaevulinate dehydratase activity of less than 5 percent of normal in the patient and activities between 26 and 51 percent of normal in the parents, in both grandfathers and in a sibling. The patient excreted large amounts of 5aminolaevulinate and coproporphyrin in the urine. Immunochemical data indicated that the enzyme deficiency in the child was associated with the production of a catalytically abnormal enzyme protein (16). The genetic defects in the patient were identified and the condition was found to be connected with two different mutations in the aminolaevulinate dehydratase gene, a paternal $823 \mathrm{G}$ to $\mathrm{A}$ transition giving rise to methionine for valine 273 and a maternal $397 \mathrm{G}$ to A transition, resulting in the substitution of arginine for glycine 133 at the carboxyl end of the zinc binding site of the enzyme (17).

Symptoms of acute porphyria were present even from birth. Severe muscular hypotonia and shallow respiration were observed, and the patient needed ventilatory support for five weeks. EMG revealed an absence of normal motor responses. After two months the child was discharged, clinically improved but without a firm diagnosis. At one year of age some hypotonia persisted but the child could sit steadily, roll around and had a good pincette grip. Weight gain was satisfactory, but feeding problems were reported. At about two years of age the child's conditon started to deteriorate. He showed signs of irritability, refused oral intake and developed progressive muscular weakness of both legs which seemed to hurt on touching. Ventilatory support again became necessary. At that time the diagnosis of aminolaevulinate dehydratase deficiency porphyria was established. During the following four and a half years several porphyric crises occurred with muscular hypotonia, respiratory distress, anorexia and painful paresthesia. Attemps to treat the crises by conventional means were generally unsuccessful. Continuous total parenteral nutrition had to be instituted because of persistent anorexia and vomiting on attempts at oral or enteral feeding. Pneumonia and septicaemia occurred frequently. Progressive muscular atrophy was seen and there was permanent partial phrenic paralysis with impaired respiratory movements and more or less constant lower pulmonary lobe atelectasis. Serum bilirubin, albumin and aminotransferase values were normal and the patient had no symptoms of liver insufficiency. With the aim of reducing the metabolic disturbance and possibly stopping the progression of the disease, a hepatic transplantation was decided upon.

\section{Transplantation and Subsequent Clinical Course}

The liver transplantation was performed at Huddinge Hospital in Stockholm in December, 1989, when the patient was six years and eight months old.

No technical difficulties or complications were encountered during the anaesthesia or the operation. The macroscopic appearance of the patient's own liver was normal although the left lobe was atrophic. Microscopic examination of the specimen revealed a normal liver, except for mild portal fibrosis. There were also some small areas with degenerated or necrotic hepatocytes. A perfectly size-matched liver from an ABO-blood group identical donor was used.

Because of $950 \mathrm{ml}$ blood loss, $600 \mathrm{ml}$ erythrocyte concentrate was given peroperatively together with plasma and buffer.

In figure 1 , the two-month postoperative period is described. The 24 hour 5 -aminolaevulinate excretion as well as the symptoms of the patient, the various medications given and signs of infection are shown in the diagram.

An episode of Pseudomonas bacteriuria was treated with piperacillin and tobramycin. Serum aminotransferase activities normalized after an initial mild ischaemic injury. In the second week, the serum aminotransferases and bilirubin increased but they returned to normal within some days. The patient was mobilized and tolerated physiotherapy. Parenteral nutrition was changed to enteral nutrition via a nasojejunal tube.

On the 25th day after the operation, the child suddenly developed severe seizures. The breathing became rapid and superficial, the heart rate rose to $150-160$ beats per minute, the central venous pressure was $7.5 \mathrm{~mm} \mathrm{Hg}$ and the diastolic systemic blood pressure $115-120 \mathrm{~mm} \mathrm{Hg}$. The stomach and intestines were dilated. Liver function was stable, but hypona- 


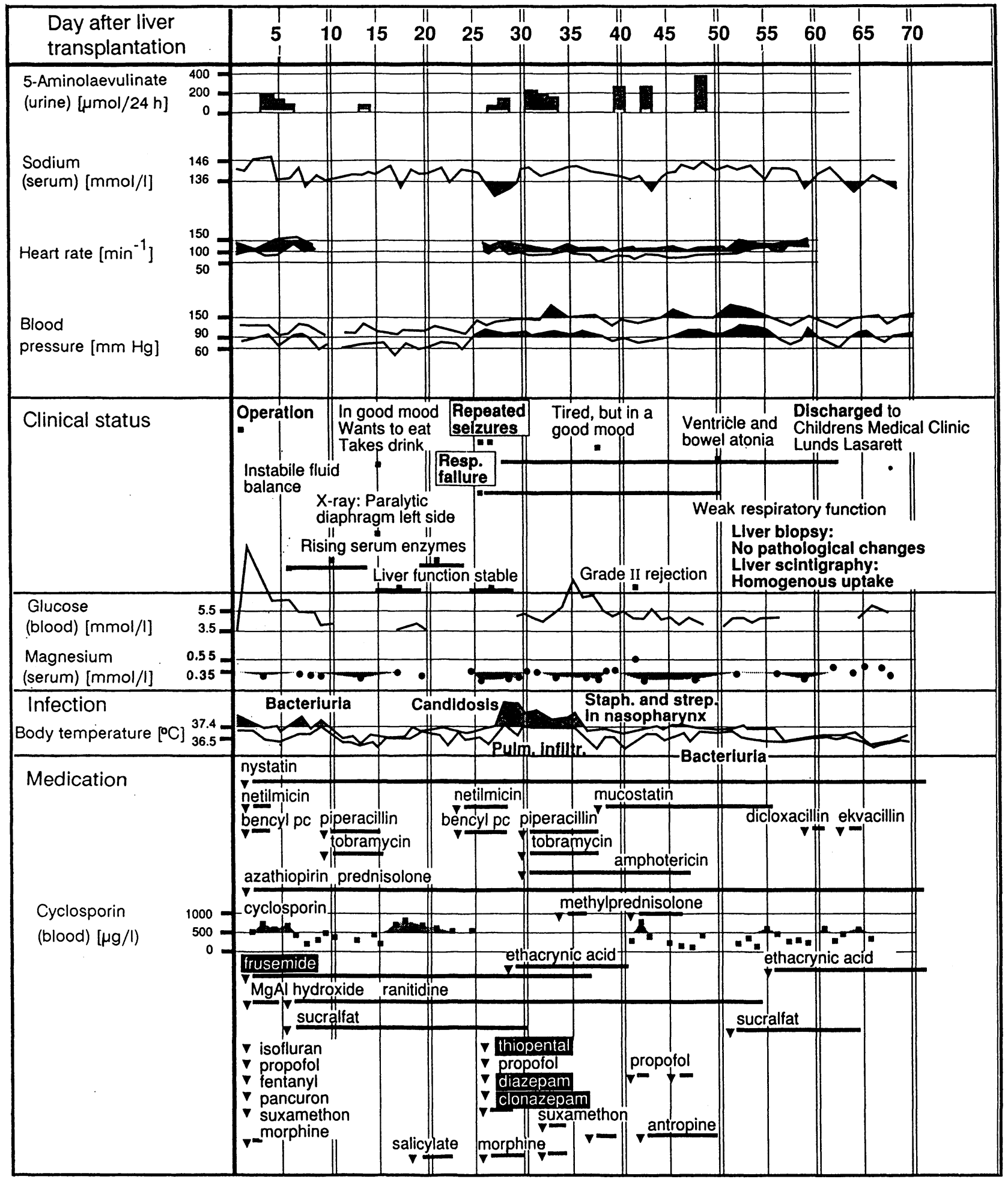

Fig. 1. Urinary 5-aminolaevulinate 24 hour excretion, clinical status, signs of infection and initiation and termination of medications during the six-month postoperative period.

Shaded areas in the diagrams represent values above or below the normal. Substances written in white on a black background are reported to be porphyrinogenic.

traemia, $130 \mathrm{mmol} / \mathrm{l}$, was present (fig. 1). During a 48-hour period at least three additional epileptiform crises occurred. Diazepam, clonazepam, thiopental, morphine and propofol were used for sedation. Pulmonary infiltrates, probably secondary to aspiration, were treated with benzylpenicillin and netilmicin. Rising serum titres against Candida antigen prompted medication with amphotericin. The patient slowly improved. After 25 days on a ventilator he was extubated but he still had poor respiratory function, attacks of bradycardia, periods of increased blood pressure and, on some occasions, hyponatraemia. Mycostatin, netilmicin, dicloxacillin and cloxacillin were used as therapy for pneumonia. After approxi- 
mately six weeks, a grade II graft rejection was diagnosed by biopsy and was successfully treated with methylprednisolone. Scintigraphy and liver biopsy 10 weeks after the transplantation showed a homogeneous uptake and no histopathological changes.

The patient was transferred to the University Hospital Lund, in March 1990. Eleven weeks after the operation his neuromuscular function deteriorated. Paralysis became total and included his respiration. He again needed ventilatory support. The course was complicated by recurrent episodes of pneumonia with atelectases, which were treated with bronchial lavage and antibiotics. An EMG showed a total absence of motor and sensory responses in the peripheral nerves and phrenic nerve. After 3 months some spontaneous diaphragmatic movements returned and he was extubated. The respiration was still not adequate, as indicated by carbon dioxide retention. To counteract atelectasis he was treated for a further 6 months with assisted ventilation at night and his respiratory function slowly stabilized. A Witzel fistula was inserted and a tube was placed in the jejunum and used for enteral feeding. All attempts at gastric feeding were unsuccessful and led immediately to gastric retention. The patient was maintained on small doses of cyclosporin, prednisolone and azathioprine and no further signs of rejection of the liver graft were seen.

The patient's neuromuscular status at present, two and a half years after the transplantation, is about the same as before the liver exchange. He has some function in his arms, although motility and strength are reduced. His hands are held in volar flexion with a decreased ability to make dorsal movements. Contractures of the ankles and knees are present. The patient can stand with support but is otherwise confined to his electric wheelchair, which he operates himself. A few crises have oc-
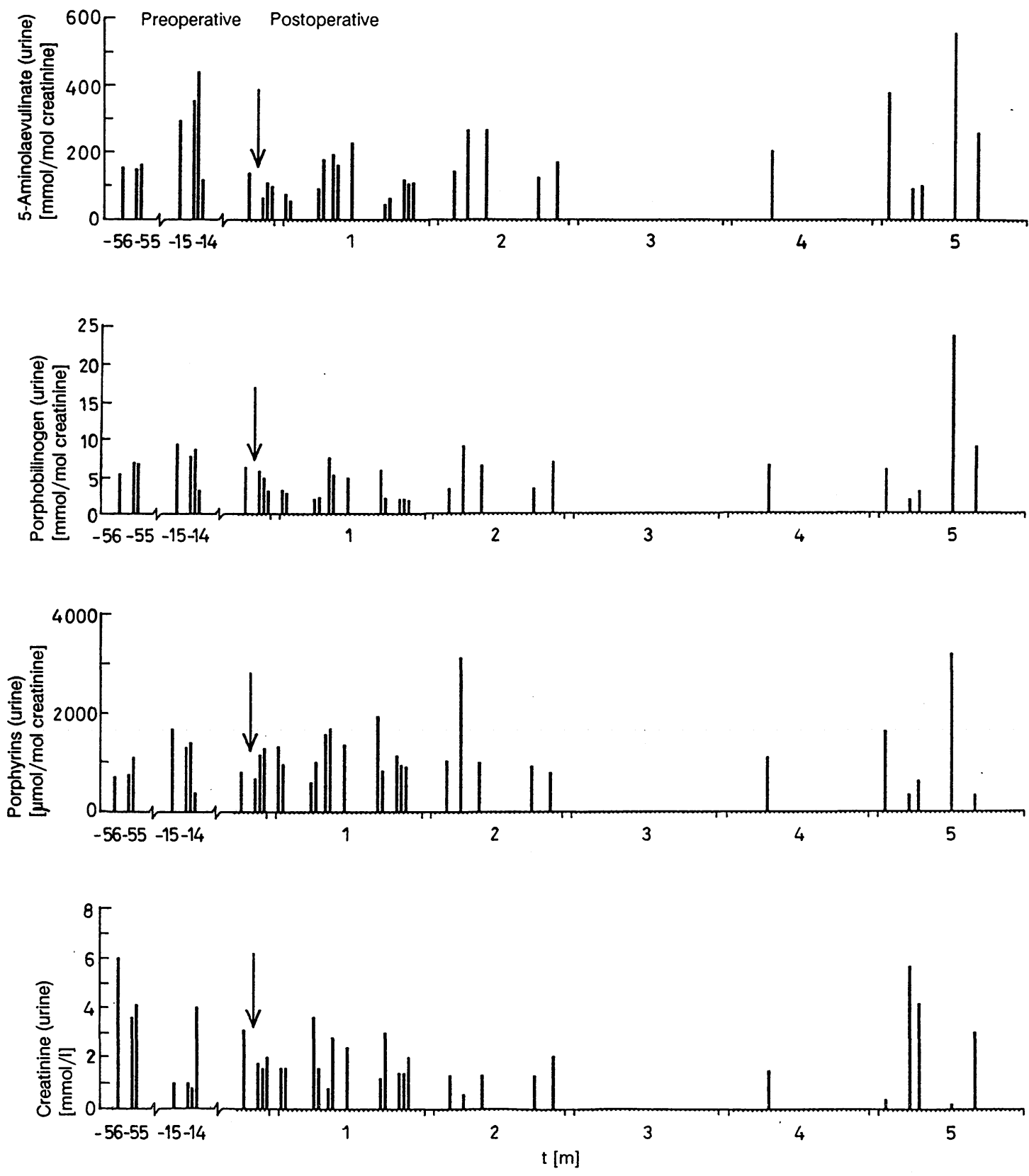

Fig. 2. Concentrations in urine of 5-aminolaevulinic acid, porphobilinogen, porphyrin and creatinine, before and after the liver transplantation.

Arrow indicates the day of transplantation. 
curred, characterized by irritative modes, obstipation, generalized pains and respiratory distress. Two major exacerbations took place in connection with a respiratory tract infection and an operation for appendicitis, respectively. The patient is mentally normal and has started school at the regular time. $\mathrm{He}$ is no longer in need for hospitalisation except during periods of crises.

\section{Biochemical Findings}

Figures 2-5 show the porphyrin and porphyrin precursor excretion in urine and faeces as well as the values for erythrocyte porphyrin and erythrocyte porphobilinogen deaminase and aminolaevulinate dehydratase activities, before and after the liver transplantation. The persistence of porphyrin and porphyrin precursor excretion in urine after the liver exchange is a striking and unexpected finding. The concentrations varied from day to day in virtually the same way as before the operation. There is a tendency for 5-aminolaevulinate and porphobilinogen concentrations to increase with time. This would not have been expected if the urinary excretion in the immediate postoperative period represented only the excretion of previously accumulated material. It is noteworthy that the seizures on the 25 th postoperative day were not preceded by any increase in the excretion of 5 aminolaevulinate and indeed, during the next few days they were followed by a steep fall in precursor excretion. On the second and third postoperative days the urinary porphyrin profiles showed a predominance of 5-8 carboxylated moieties which was probably due to an impaired decarboxylation activity in the parenchyma of a not yet stabilized graft (fig. 3). From the fourth day on, the excretion profiles did not differ significantly from those found two days before the operation. After the liver transplantation the amounts of porphyrin excreted in faeces considerably decreased, compared with the preoperative state, from
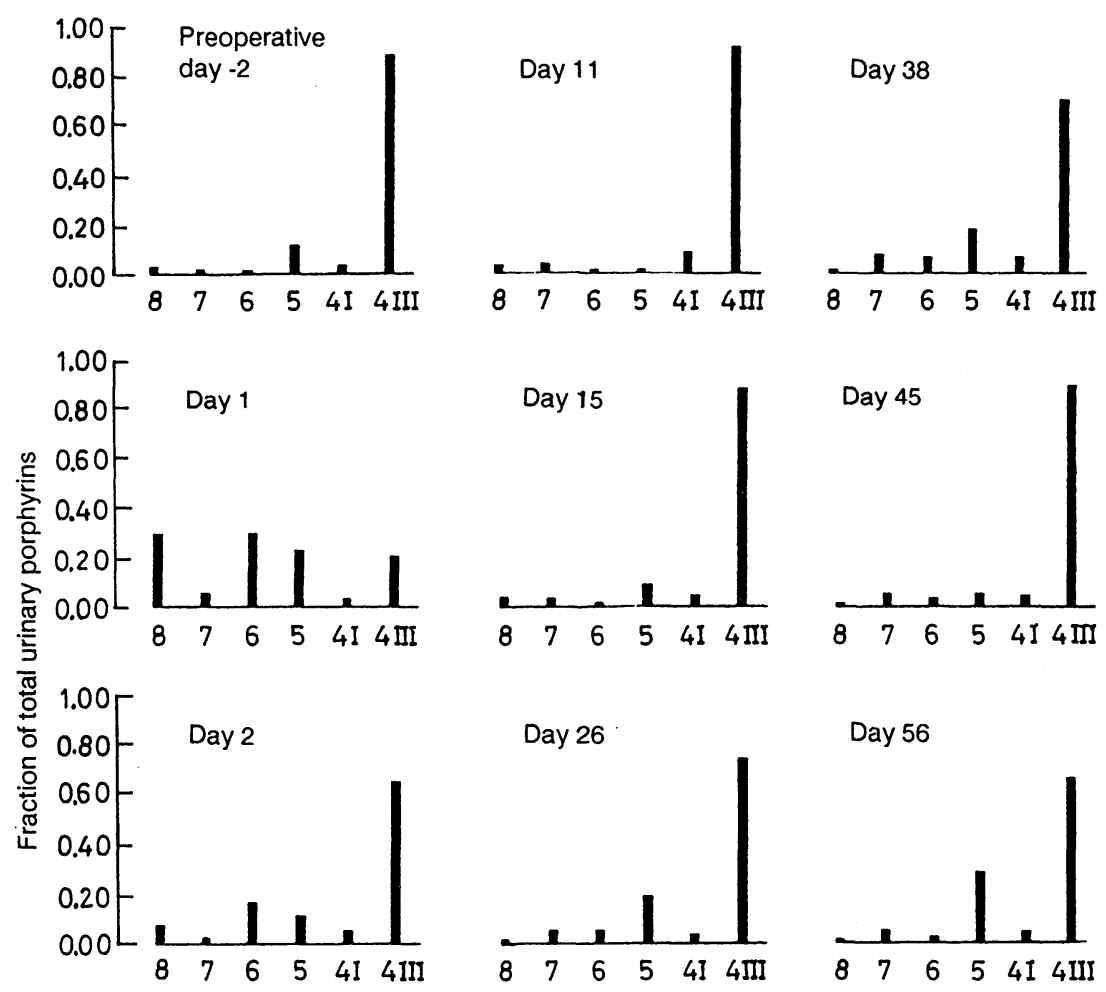

Day 56
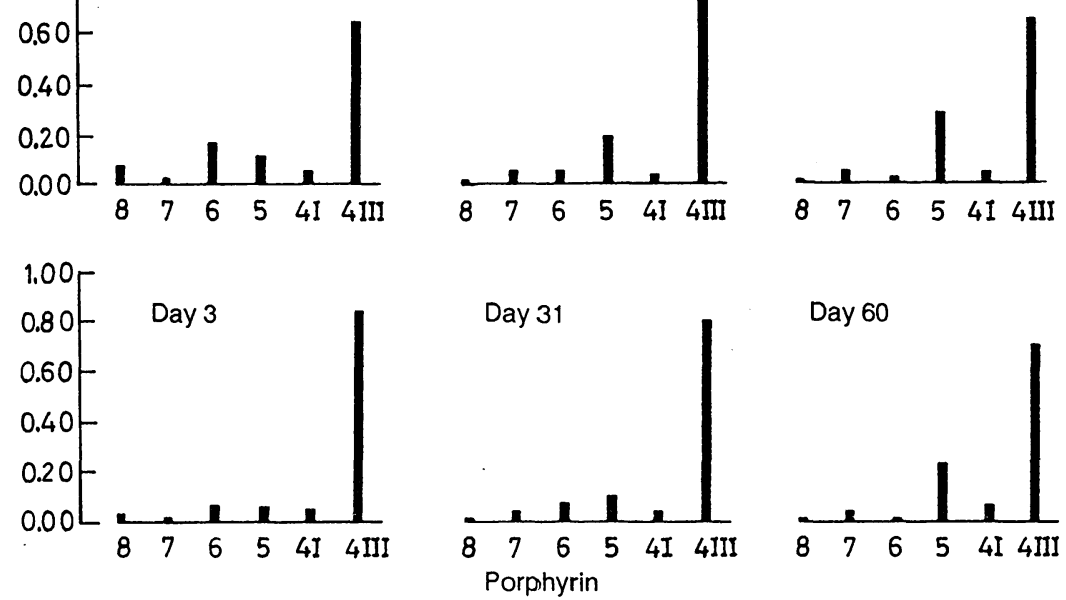

Day 60

Fig. 3. Urinary porphyrin excretion patterns 2 days before and varying numbers of days after the liver transplantation. $8,7,6,5,4$ : number of porphyrin carboxylate side groups

$4 \mathrm{I}$ : coproporphyrin I isomer

4 III: coproporphyrin III isomer 


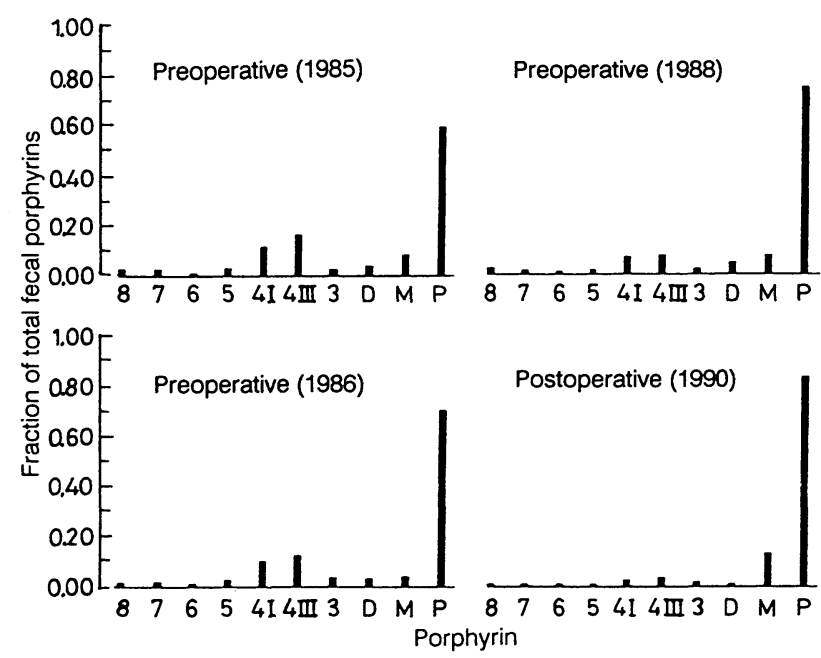

Fig. 4. Faecal porphyrin excretion patterns on three occasions before, and 6 days after the liver transplantation. $8,7,6,5,4,3$ : number of carboxylate side-chains $4 \mathrm{I}$ : coproporphyrin I-isomer

4 III: coproporphyrin III-isomer

D: deuteroporphyrin

M: mesoporphyrin

P: protoporphyrin

$462 \mathrm{nmol} / \mathrm{g}$ to $7 \mathrm{nmol} / \mathrm{g}$. The porphyrin pattern, however, was unchanged, with 80 per cent protoporphyrin and a coproporphyrin III/I isomer ratio larger than unity. In the early postoperative period the values for the red blood cell quantities were presumably influenced by the large volume of erythrocytes given to the patient. Thus, due to transfusions of normal donor blood, erythrocyte aminolaevulinate dehydratase activity was normalised postoperatively. Assays performed one year after the transplantation showed a regression in activity to the preoperative level.

Biochemical investigation of the liver explant was not performed.

\section{Discussion}

Experience of the clinical features of severe aminolaevulinate dehydratase deficiency porphyria is limited. The symptoms, however, seem to agree with those known to occur in the other forms of acute porphyria, as well as those seen in tyrosinaemia. When evaluating the outcome of therapeutic measures in this condition, attention therefore should be focused on symptoms such as pain, paralysis, mental disturbance, seizures, respiratory distress, vomiting, ileus, tachycardia, hypertension and hyponatraemia (11, 18). With the exception of seizures, all the symptoms mentioned were part of the patient's preoperative porphyric attacks. However, even when occurring in
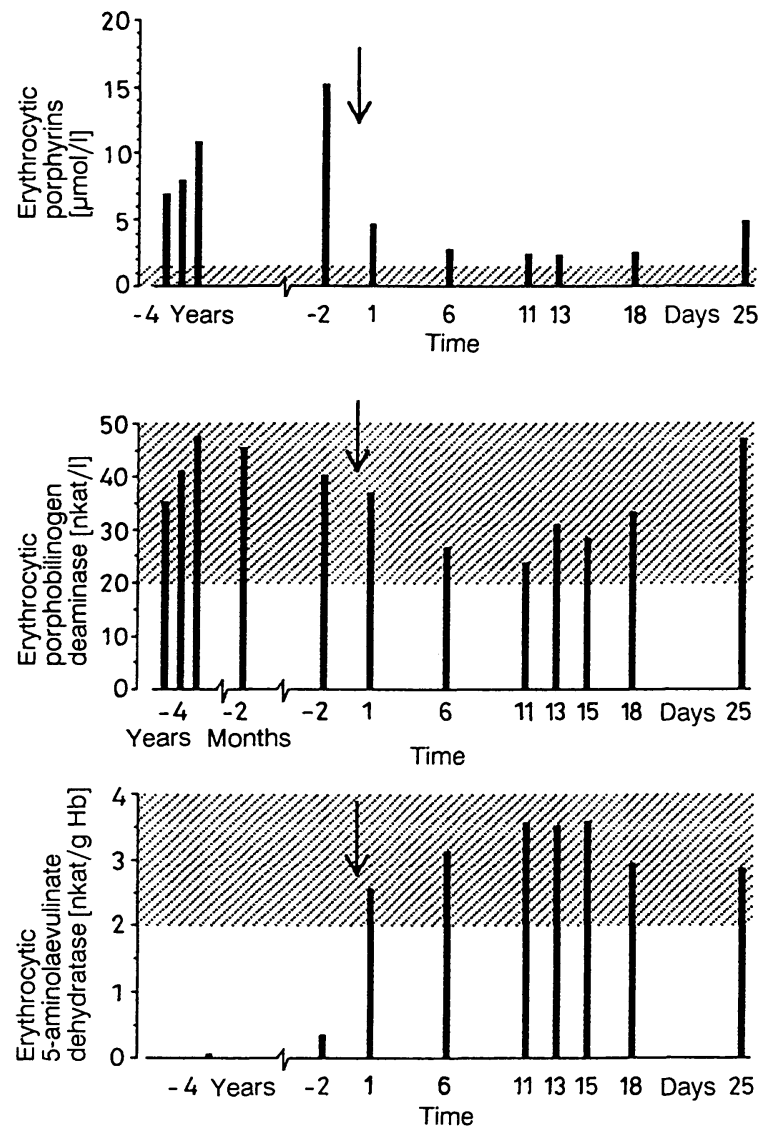

Fig. 5. Erythrocyte porphyrin concentration and erythrocyte porphobilinogen deaminase and aminolaevulinate dehydratase activities before and after the liver transplantation.

clusters, the symptoms are not specific for porphyria, especially not in a postoperative clinical setting, and the absence of porphyric symptoms cannot be viewed as proof that correction of the metabolic defect has taken place. On the other hand, if the symptoms are found to coincide with the occurrence of a porphyrinogenic factor, they are more likely to be due to the basic disease. During a porphyric or tyrosinaemic crisis the 5-aminolaevulinate excretion is, as a rule, higher than normal, even if there is no correlation between the magnitude of the excretion and the severity of the symptoms (18). At least in tyrosinaemia, a neurogenic crisis is not invariably accompanied by further increases in porphyrin precursor excretion. Nevertheless, if a clinical manifestation of porphyria is accompanied by an augmented excretion of 5-aminolaevulinate, this supports the diagnosis of a porphyric attack.

On the 25th postoperative day, symptoms suggestive of an attack of acute porphyria developed in our patient. Coinciding with the appearance of pulmonary infiltrates and a rising serum titre of Candida antigen, 
and after a period of high blood concentrations of the porphyrinogenic agent, cyclosporin $(19,20)$, the patient suddenly developed repeated attacks of epileptiform seizures and respiratory failure as well as sinus tachycardia, diastolic hypertension and hyponatraemia. The respiratory distress, however, could be explained by the pulmonary infection, in combination with persistent phrenic paralysis, as observed preoperatively. Furthermore, seizures are not common in porphyria and were not a part of the preoperative porphyric symptomatology of the patient. On the other hand, neurological complications are frequently observed postoperatively in transplantation patients and symptoms may persist for months $(21-26)$. Cyclosporin is probably involved since approximately 10 per cent of patients receiving the drug develop seizures (27). A number of additional factors may be of importance e. g., fluid retention, hypertension, hyponatraemia, hypomagnesaemia, hyperosmolarity, prolonged ranitidine medication, high doses of steroids and graft dysfunction (28). The concentration of magnesium in blood seems to be of critical significance $(23,29,30)$. As seen in figure 1 , during the week preceding the neurological crisis, on the 25 th postoperative day, toxic blood concentrations of cyclosporin were recorded as well as concentrations of serum magnesium two standard deviations below the normal mean. In view of this and the facts that diastolic and systolic hypertension were present already on the day before the onset of the seizures and that the 5-aminolaevulinate excretion did not increase in connection with the attack, the overall clinical and biochemical picture was interpreted as not being due to induction of a latent porphyric condition, but as secondary to cyclosporin neurotoxicity in combination with hypomagnesaemia and a chronically impaired pulmonary function, aggravated by pneumonia.

The second polyneuropathic crisis, which occurred about 11 weeks after the transplantation, was less acute in onset, but considerably more profound and it persisted longer.

The onset and progress of the attacks were not accompanied by any significant changes in porphyrin precursor excretion. Apart from the barbiturate and benzodiazepine administration 5 weeks before and an instance of bacteriuria 3 weeks prior to the crisis, no factor known to trigger an attack of porphyria could be identified. Values for serum cyclosporin and magnesium concentrations are not available for the time of deterioration, but in the preceding week borderline and/or toxic values were repeatedly recorded for cyclosporin, and serum magnesium concentrations were somewhat low. It is known that liver transplant patients with cyclosporin-provoked neuropathy may develop hemiplegia or tetraplegia $(26,28)$. In two cases a spinal cord syndrome developed a considerable period after an attack of seizures, as in our patient. Although the generalized polyneuropathy in our patient was indistinguishable from similar episodes before the transplantation, the possibility cannot be excluded that cyclosporin contributed to this neurotoxic attack. Unaltered porphyrin precursor excretion gives some support for such a conclusion.

The postoperative clinical, pharmacological and biochemical patterns were interactive and complex, and it is very difficult to evaluate effects of the liver transplant on the porphyric condition. After the two early crises the patient has had a few further attacks. However, he feels better than before, can spend his time at home, and is no longer in need of total parenteral nutrition. As a result of transfusions of normal donor blood, erythrocyte aminolaevulinate dehydratase activity normalised in the patient (fig. 5). Assays performed 12 months after the operation showed a regression of enzyme activity to the preoperative level. An effect of the liver exchange on the erythroid aminolaevulinate dehydratase gene expression, was thus not observed.

The exchange of the aminolaevulinate dehydratasedeficient liver for a normal organ did not affect the high basal urinary excretion of porphyrins and porphyrin precursors, and these metabolites obviously originate mainly from extrahepatic tissues that are still affected by aminolaevulinate dehydratase deficiency. The fact that the patient after the transplantation is able to withstand severe porphyrinogenic challenges, such as barbiturate medication, without developing polyneuropathy, and without increasing the 5-aminolaevulinate and porphobilinogen excretion, indicates that the excess production of metabolites takes place in cells that are not under the control of the aminolaevulinate dehydratase-inductive mechanisms responsible for the activation of the disease by such agents. The erythron is the major reservoir for such cells in the body and it seems that a more profound metabolic effect might be achieved by bone marrow transplantation. Unfortunately, this approach is not possible because of the lack of a suitable sibling donor.

\section{Acknowledgement}

The skilfull technical assistance provided by laboratory technologists Agneta Liljegren, Maria Kobak and Gunilla Larsson is gratefully acknowledged. 


\section{References}

1. Bird, T. D., Hamernyik, P., Nutter, J. Y. \& Labbe, R. F. (1979) Inherited deficiency of delta-aminolaevulinic acid dehydratase. Am. J. Hum. Genet. 31, 662-668.

2. Doss, M., von Tiepermann, R., Schneider, J. \& Schmid, H. (1979) New type of hepatic porphyria with porphobilinogen synthase defect and intermittent acute clinical manifestation. Klin. Wochenschr. 57, 1123-1127.

3. Doss, M., von Tiepermann, R. \& Schneider, J. (1980) Acute hepatic porphyria syndrome with porphobilinogen synthase defect. Int. J. Biochem. 12, 823-826.

4. Thunell, S., Holmberg, L. \& Lundgren, J. (1987) Aminolaevulinate dehydratase porphyria in infancy. A clinical and biochemical study. J. Clin. Chem. Clin. Biochem. 25, 5-14.

5. Hassoun, A., Mercelis, R., Verstraeten, L., De Bock, R. \& Martin, J. J. (1989) Porphyric neuropathy and hereditary delta-aminolevulinic acid dehydratase deficiency in an adult. Neurol. Sci. 95, 39-47.

6. Becker, D. M., Viljoen, D. \& Kramer, S. (1976) Porphyrin precursors and their effect in vitro in some aspects of nerve function. In: Porphyrins in Human Disease (Doss, M., ed.) Karger, Basel, pp. 163-172.

7. Bornstein, J. C., Picket, J. B. \& Diamond, I. (1979) Inhibition of the evoked release of acetylcholine by the porphyrin precursor, delta-aminolaevulinic acid. Ann. Neurol. 5, 94-96.

8. Brennan, M. J. W., Cantrill, R. C. \& Kramer, S. (1980) Effect of delta-aminolaevulinic acid and GABA receptorbinding in synaptic plasma membranes. Int. J. Biochem. $12,833-835$

9. Percy, U. A., Lamm, M. C. L. \& Taljaard, J. J. F. (1981) Delta-aminolaevulinic acid uptake, toxicity and effect on $\left[{ }^{14} \mathrm{C}\right] 5$-aminobutyric acid uptake into neurons and glia in culture. J. Neurochem. 36, 69-76.

10. Cutler, M. G., Moore, M. R. \& Dick, J. M. (1985) Pharmacological effects of delta-aminolaevulinic acid and gamma-aminobutyric acid in isolated preparations of rabbit jejunum. Br. J. Pharmacol. 86, 680-686.

11. Kappas, A., Sassa, S., Galbraith, R. A. \& Nordmann, Y. (1989) The Metabolic Basis of Inherited Disease (Scriver, C. R., Beaudet, A. L., Sly, W. S. \& Valle, D., eds.) 6th ed., pp. 1305-1365. McGraw-Hill.

12. Starzl, T. E., Bilöheimer, D. W., Bahnson, H. T., Shaw, Jr. B. W., Hardesty, R. L., Griffith, B. P., Iwatsuki, S., Zitelli, B. J., Gaartner, Jr. J. C., Malatack, J. J. \& Urbach, A. H. (1984) Heart-liver transplantation in a patient with familial hypercholesterolaemia. Lancet 1, 1382-1383.

13. Samuel, D., Boboc, B., Bernuau, J., Bismuth, H. \& Benhamou, J-P. (1988) Liver transplantation for protoporphyria. Gastroenterology 95, 816-819.

14. Polson, R. J., Chang, K. L., Rolles, K., Calne, R. Y. \& Williams, R. (1988) The effect of liver transplantation in a 13-year-old boy with erythropoietic protoporphyria. Transplantation 46, 386-388.

15. Bloomer, J. R., Weimer, M. K., Bossenmaier, I. C., Snower, D. C., Payne, W. D. \& Ascher, N. L. (1989) Liver transplantation in a patient with protoporphyria. Gastroenterology 97, 188-194.
16. Fujita, H., Sassa, S., Lundgren, J., Holmberg, L., Thunell, S. \& Kappas, A. (1987) Enzymatic defect in a child with hepatic porphyria due to homozygous delta-aminolevulinic acid dehydratase deficiency. Immunochemical studies. Pediatrics $80,880-885$.

17. Plewinska, M., Thunell, S., Holmberg, L., Wetmur, J. G. \& Desnick, R. J. (1991) Delta-aminolevulinate dehydratase deficient porphyria: Identification of the molecular lesions in a severely affected homozygote. Amer. J. Hum. Genet., in press.

18. Mitchell, G., Larochele, J., Lambert, M., Michand, J., Grenier, A., Ogier, H., Gauthier, M., Lacroix, J., Vanasse, M., Labrisseau, A., Paradis, K., Weber, A., Lefeure, Y., Melancon, S. \& Dallaire, L. (1990) Neurologic crisis in hereditary tyrosinemia. N. Engl. J. Med. 322, 432-437.

19. Moore, M. R., McColl, K. E., Rimington, C. \& Goldberg, A. (1987) Pathogenesis of neuropathy of acute porphyria. In: Disorders of Porphyrin Metabolism, pp. 119-137. Plenum Medic.

20. Moore, M. R., Thompson, G. C. \& McColl, K. E. (1987) The effects of some chemotherapeutic and immunosuppresive agents on 5-aminolaevulinate synthase.

21. Joss, D. V., Barrett, A. J., Kendra, J. R., Lucas, C. F. \& Desai, S. (1982) Hypertension and convulsions in children receiving cyclosporin A. Lancet $I, 906$.

22. Durrant, S., Chipping, P. M., Palmer, S. \& Gordon-Smith, E. C. (1982) Cyclosporin A, methylprednisolone and convulsions. Lancet $I I, 829-830$.

23. Allen, R. D., Hunnisett, A. G. \& Morris, P. J. (1985) Cyclosporin and magnesium. Lancet $1,1283-1284$

24. Nordal, K. P., Talseth, T., Dahl, E., Attramadal, A., Albrechtsen, D., Halse, J., Brodwall, E. K. \& Flatmark, A. (1985) Aluminium overload, a predisposing condition for epileptic seizures in renal transplant patients treated with cyclosporin. Lancet $I I, 153-154$.

25. De Groen, P. C., Aksamit, A. J., Rakela, J., Forbes, G. S. \& Krom, R. A. (1987) Central nervous system toxicity after liver transplantation. The role of cyclosporine and cholesterol. N. Engl. J. Med. 317, 861 - 866.

26. De Groen, P. C., Aksamit, A. J., Rakela, J., Forbes, G. S. \& Krom, R. A. F. (1988) The role of cyclosporin A and cholesterol in a new central nervous system syndrome after liver transplantation. Transpl. Proc. $X X, 625-627$.

27. Kahan, B. D., Flechner, S. M., Lorber, M. I., Jensen, C., Golden, D. \& Van Buren, C. T. (1986) Complications of cyclosporin therapy. World J. Surg. 10, 348-360.

28. Voigt, D. P., Lederman, R. J., Carey, W. D. \& Broughan, T. A. (1988) Neurologic complications of liver transplantation. Transplantation 6, 1057-1061.

29. Thompson, C. B., June, C. H., Sullivan, K. M. \& Thomas, E. D. (1984) Association between cyclosporin neurotoxicity and hypomagnesemia. Lancet II, 1116-1120.

30. Adams, D. H., Ponsford, S., Gunson, B., Boon, A., Honigsberger, L., Williams, A., Buckels, J., Elias, E. \& McMaster, P. (1987) Neurologic complications following liver transplantation. Lancet I, 949-951.

Stig Thunell, MD, PhD

Department of Clinical Chemistry

St. Göran's Hospital

S-11281 Stockholm 